\title{
MODELO MATEMÁTICO DO ESCOAMENTO GASOSO E TROCAS TÉRMICAS NO PROCESSO DE CARVOEJAMENTO EM FORNO RETANGULAR*
}

Jayme Alex Marques da Silva' Jose Adilson de Castro ${ }^{2}$

\section{Resumo}

A utilização de carvão vegetal na fabricação do ferro-gusa tem ganhado destaque devido a suas vantagens ambientais em comparação ao coque. A necessidade de suprir a demanda de consumo de carvão vegetal pelas siderurgias faz com que os produtores busquem maior produtividade e qualidade através da busca constante na redução do tempo de produção do carvão. O presente trabalho apresenta 0 desenvolvimento de um modelo matemático tri-dimensional transiente, para simulação da secagem, carbonização e resfriamento no interior de um forno retangular de alvenaria. Foi desenvolvido um modelo acoplado baseado na solução das equações de conservação da quantidade de movimento, energia e espécies químicas. As soluções das equações diferenciais que representam os fenômenos são obtidas através da técnica de volumes finitos. Ao longo do processo de carvoejamento, o modelo considera a transferência de massa e calor associada à cinética de reação de cada componente. O modelo é tridimensional e transiente, sendo capaz de prever detalhes dos fenômenos no interior do forno e sua evolução temporal o que possibilita o controle das fases de fabricação do carvão vegetal.

Palavras-chave: Modelo matemático; Carbonização; Secagem; Carvão vegetal.

\section{MATHEMATICAL MODELING OF GAS FLOW AND HEAT TRANSFER IN THE RETANGULAR FURNACE FOR CHARCOAL PRODUCTION}

\section{Abstract}

The utilization of charcoal in the production of hot metal has environmental advantages when in comparison with coke. The need of supplying the demand of charcoal from the steelworks implies that the producers seek productivity increasing through the minimization of the charcoal production time and keeping the same quality. The present work presents a transient and three dimensional numerical modeling to simulate the drying process, carbonization and cooling inside a rectangular brickwork furnace of charcoal production. A mathematical model utilizing the conservation equations of momentum, energy and chemical species was made and the solution of the corresponding differential equations were discretized by the finite volume method. The modeling has considered mass and heat transfer coupled with kinetic reaction of each component, being capable to simulate details of the physical phenomena inside the furnace and calculate the main properties over time processing, allowing the understanding of how to control the charcoal production during the manufacturing stages.

Keywords: Mathematical model; Carbonization; Drying; Charcoal.

1 Engenheiro Mecânico, Mestre em engenharia Metalúrgica, Votorantin Siderurgia, Barra Mansa, RJ, Brasil.

2 Ph.D. Professor, Universidade Federal Fluminense (UFF), Volta Redonda, RJ, Brasil.

* Contribuição técnica ao 44ํ Seminário de Redução de Minério de Ferro e Matérias-primas, 15 Simpósio Brasileiro de Minério de Ferro e 20 ${ }^{\circ} 5_{2}$ impósio Brasileiro de Aglomeração de Minério de Ferro, 15 a 18 de setembro de 2014, Belo Horizonte, MG, Brasil. 


\section{INTRODUÇÃO}

Recentemente, a siderurgia brasileira têm direcionado esforços no sentido de se utilizar apenas carvão vegetal de florestas plantadas. Neste sentido, os grandes produtores utilizam-se de florestas de eucaliptos. Entretanto, grande parte da produção de carvão vegetal no Brasil, é proveniente de forno de baixa eficiência, relacionada muitas vezes à falta de controle das variáveis de processo de carvoejamento e não utilização dos subprodutos provenientes da pirólise. Do ponto de vista da utilização dos equipamentos, as etapas de secagem e resfriamento representam os gargalos do processo, consumindo mais de $80 \%$ do tempo total do ciclo da produção em fornos retangulares de alvenaria, tipo o forno RAC 220 $\left(\mathrm{R}=\right.$ Retangular; $\mathrm{AC}=$ Acesita; Capacidade $\left.=220 \mathrm{~m}^{3}\right)$. Neste estudo, foi, portanto, dado um enfoque prioritário nestas etapas visando o desenvolvimento de um procedimento operacional que otimize estas etapas, possibilitando uma disponibilidade maior dos fornos. A abordagem foi direcionada à formulação de um modelo matemático detalhado do ciclo completo de produção de carvão em fornos de grande volume interno fabricado de alvenaria e identificando as etapas criticas do processo em termos de trocas térmicas. Alguns modelos visando o estudo das etapas de resfriamento propondo tecnologias para redução do tempo total de produção de carvão tem sido apresentado, por exemplo Raad et al.[1] propuseram um modelo que visa calcular a cinética da carbonização e transferência de calor. Entretanto, um modelo detalhado considerando o escoamento gasoso, tridimensional e transiente e com detalhamento dos fenômenos ainda precisa ser desenvolvido. Neste trabalho, procura-se preencher esta lacuna e abrir a possibilidade para a inclusão de modelos cinéticos acoplados aos fenômenos fluidodinâmicos e de transferência de calor. Neste contexto, o presente trabalho apresenta um modelo baseado no conceito de múltiplas fases e multicomponente, tridimensional e transiente capaz de simular o escoamento, trocas térmicas e transferência de massa associadas à cinética química envolvendo as espécies químicas em um reator retangular construído de alvenaria [2]. Visando obter tempos totais de produção de carvão menores, propõe-se uma modificação no teto (cobertura) do forno que contribui de forma significativa nas etapas de aquecimento e secagem e no resfriamento acelerado induzido por convecção devido a gradientes térmicos.

\section{METODOLOGIA}

Os fornos de carvoejamento utilizados para testar o modelo matemático foram construídos em alvenaria. Um dos fornos possui teto em alvenaria, conforme modelo original e outro foi modificado introduzindo-se os painéis articulados no teto. Para os dois modelos foram mantidos as dimensões de $39 \mathrm{~m} \times 4 \mathrm{~m} \times 4,7 \mathrm{~m}$. Os dados do processo foram obtidos através do levantamento de campo, utilizando sensores de temperatura, medidores de vazão, entre outros.

\subsection{Modelo Matemático}

O modelo matemático é composto pelas equações de momentum, energia e conservação de espécies químicas nas fases correspondentes. São acopladas por relações semi-empíricas que determinam as taxas de transferências de momentum, e massa e correspondentes trocas térmicas. As Equações (1) a (6) traduzem de

* Contribuição técnica ao 44ํㅗㄴ Seminário de Redução de Minério de Ferro e Matérias-primas, 15ㅇ Simpósio Brasileiro de Minério de Ferro e ${ }^{2}{ }_{853}$ Simpósio Brasileiro de Aglomeração de Minério de Ferro, 15 a 18 de setembro de 2014, Belo Horizonte, MG, Brasil. 
forma compacta estas relações que constituem a essência do modelo matemático considerado neste estudo [2].

$$
\begin{aligned}
& \frac{\partial\left(\rho_{i} \varepsilon_{i} u_{i, j}\right)}{\partial t}+\frac{\partial\left(\rho_{i} \varepsilon_{i} u_{i, k} u_{i, j}\right)}{\partial x_{k}}=\frac{\partial}{\partial x_{k}}\left(\mu_{i} \frac{\partial u_{i, j}}{\partial x_{k}}\right)-\frac{\partial P_{i}}{\partial x_{j}}-F_{j}^{i-l}+\rho_{i} \varepsilon_{i} g_{j} \\
& \frac{\partial\left(\rho_{i} \varepsilon_{i}\right)}{\partial t}+\frac{\partial\left(\rho_{i} \varepsilon_{i} u_{i, k}\right)}{\partial x_{k}}=\sum_{m=1}^{\text {Nreacts }} M_{n} r_{m} \\
& \frac{\partial\left(\rho_{i} \varepsilon_{i} H_{i}\right)}{\partial t}+\frac{\partial\left(\rho_{i} \varepsilon_{i} u_{i, k} H_{i}\right)}{\partial x_{k}}=\frac{\partial}{\partial x_{k}}\left(\frac{k_{i}}{C_{p_{i}}} \frac{\partial H_{i}}{\partial x_{k}}\right)+E^{i-l}+\sum_{m=1}^{\text {Nreacts }} \Delta H_{m} r_{m} \\
& \frac{\partial\left(\rho_{i} \varepsilon_{i} \phi_{n}\right)}{\partial t}+\frac{\partial\left(\rho_{i} \varepsilon_{i} u_{i, k} \phi_{n}\right)}{\partial x_{k}}=\frac{\partial}{\partial x_{k}}\left(D_{n}^{e f f} \frac{\partial \phi_{n}}{\partial x_{k}}\right)+\sum_{m=1}^{N r e a c t s} M_{n} r_{m} \\
& F_{j}^{i-l}=\left[1.75 \rho_{i}+\frac{150 \mu_{i}}{\left|\vec{U}_{i}-\vec{U}_{l}\right|}\left(\frac{\varepsilon_{l}}{d_{l} \varphi_{l}}\right)\right]\left(\frac{\varepsilon_{l}}{\left(1-\varepsilon_{l}\right)^{3} d_{l} \varphi_{l}}\right)\left|\vec{U}_{i}-\vec{U}_{l}\right|\left(u_{i, j}-u_{l, j}\right) \\
& E^{i-l}=\frac{6 \varepsilon_{l}}{d_{l} \varphi_{l}} \frac{k_{i}}{\left(d_{l} \varphi_{l}\right)}\left[2+0.39\left(\operatorname{Re}_{i-l}\right)^{1 / 2}\left(\operatorname{Pr}_{i}\right)^{1 / 3}\right]\left(T_{i}-T_{l}\right)
\end{aligned}
$$

Onde os índices $i, k$ e $/$ representam as fases consideradas (gás, sólido e condensado) enquanto o índice j representa as direções coordenadas. As variáveis $\mathrm{u}, \mathrm{H}, \mathrm{P}$ e $\mathrm{T}$ representam as componentes de velocidades, entalpia, pressão e temperaturas da fases, respectivamente. As variáveis $\Phi, \varepsilon, \varphi, \rho$ e $\mu$ representam a fração mássica da espécie química, fração volumétrica da fase, fator de forma do sólido e viscosidade efetiva da fase, respectivamente. $M$ representa o peso

\begin{tabular}{|c|c|c|}
\hline Fases & & Espécies Químicas( $\Phi)$ \\
\hline Gás & \multicolumn{2}{|c|}{$\mathrm{CO}, \mathrm{CO}_{2}, \mathrm{O}_{2}, \mathrm{H}_{2}, \mathrm{H}_{2} \mathrm{O}, \mathrm{N}_{2}, \mathrm{CH}_{4}, \mathrm{C}_{2} \mathrm{H}_{6}, \mathrm{C}_{3} \mathrm{H}_{8}, \mathrm{C}_{4} \mathrm{H}_{10}$} \\
\hline \multirow{2}{*}{ Sólido } & $\begin{array}{l}\text { Matéria } \\
\text { carbonosa }\end{array}$ & $\begin{array}{c}\left(\mathrm{C}_{6} \mathrm{H}_{10} \mathrm{O}_{5}\right) \text { n Celulose, }\left(\mathrm{C}_{10} \mathrm{H}_{12} \mathrm{O}_{4}\right) \text { Lignina, } \\
\left(\mathrm{C}_{5} \mathrm{H}_{8} \mathrm{O}_{4}\right) \text { ne }\left(\mathrm{C}_{6} \mathrm{H}_{10} \mathrm{O}_{5}\right) \text { n, Hemicelulose, voláteis }\end{array}$ \\
\hline & Cinzas & $\mathrm{SiO}_{2}, \mathrm{CaO}, \mathrm{MgO}, \mathrm{Al}_{2} \mathrm{O}_{3}, \mathrm{~S}, \mathrm{P}_{2} \mathrm{O}_{5}$ \\
\hline Líquidos & \multicolumn{2}{|c|}{$\begin{array}{l}\text { Condensáveis: extrato pirolenhoso ( ácido acético } \mathrm{CH}_{3} \mathrm{COOH}+ \\
\left.\mathrm{H}_{2} \mathrm{O}\right) \text { e alcatrão }\left(\mathrm{C}_{12} \mathrm{H}_{22}\right)+\mathrm{H}_{2} \mathrm{O}\end{array}$} \\
\hline
\end{tabular}
molecular da espécie química enquanto $r_{m}$ representa a taxa de reação envolvendo com respeito à espécie $m$. $D_{\text {eff }}$ é o coeficiente de difusão efetivo da espécie química e $d$ o diâmetro médio efetivo do sólido. A Tabela 1 apresenta as fases e espécies químicas consideradas no modelo.

Tabela 1: Fases consideradas para o modelamento matemático

\subsection{Condições Iniciais e de Contornos}

Ao modelo representado pelas Equações (1) a (6) devem ser acrescidas as condições iniciais e de contorno para as variáveis envolvidas. Para as condições

* Contribuição técnica ao $44^{\circ}$ Seminário de Redução de Minério de Ferro e Matérias-primas, 15은 Simpósio Brasileiro de Minério de Ferro e ${ }^{2}{ }_{8}^{\circ}$ Simpósio Brasileiro de Aglomeração de Minério de Ferro, 15 a 18 de setembro de 2014, Belo Horizonte, MG, Brasil. 
iniciais considera-se a lignina com $25 \%$ da composição da madeira, celulose com $45 \%$ e hemicelulose com $30 \%$ da composição principal da madeira; e seu arranjo geométrico (as toras) à temperatura ambiente. As paredes são consideradas como impermeáveis e coeficientes de trocas térmicas são calculados localmente conforme a constituição das paredes e painéis articulados. São calculados coeficientes de convecção de calor local interno e externo dependendo da etapa que se encontra o processo.

\subsection{Reações Consideradas no Modelo}

No modelo são consideradas trocas químicas devido a reações químicas e transformações de fases. A ignição inicial e admissão de ar promove a autosustentação do processo fornecendo calor para que as reações endotérmicas de decomposição da madeira sejam completadas. Os gases e vapores condensáveis geram líquidos e percolam sendo coletados em pontos determinados do reator. A cinética, das diversas reações foram adaptadas da literatura [2-6]. A Tabela 2 relaciona as principais reações consideradas no modelo. O carbono fixo e as cinzas são residuais das reações de transformação da madeira e são obtidos através da conservação de massa.

Tabela 2: Principais reações consideradas no modelo

Madeira (Celulose + Lignina + hemicelulose $)+\mathrm{O}_{2} \Rightarrow \mathrm{C}($ fixo $)+$ Cinzas $+\mathrm{CO}(\mathrm{g})$

+ Volateis + condensaveis

\begin{tabular}{|c|c|}
\hline $\mathrm{H}_{2} \mathrm{O}_{(\text {madeira })} \Rightarrow \mathrm{H}_{2} \mathrm{O}_{(v)}$ & (8) \\
\hline $\begin{aligned} \text { Voláteis(madeira) } \Rightarrow & 0,05 \mathrm{CH}_{4}(g)+0,05 \mathrm{C}_{2} \mathrm{H}_{6}(g)+0,75 \mathrm{C}_{3} \mathrm{H}_{8}(g)+0,25 \mathrm{C}_{4} \mathrm{H}_{10}(g) \\
& +0,01 \mathrm{H}_{2} \mathrm{O}_{(v)}+0,25 \mathrm{CO}_{2}(g)+0,05 \mathrm{~N}_{2}(g)\end{aligned}$ & (9) \\
\hline Voláteis (madeira) $+\alpha_{1} \mathrm{O}_{2}(g) \Rightarrow \alpha_{2} \mathrm{CO}_{2}(g)+\alpha_{3} \mathrm{H}_{2} \mathrm{O}(v)+\alpha_{4} N_{2}(g)$ & $(10)$ \\
\hline Voláteis (madeira) $+\beta_{1} C O_{2}(g) \Rightarrow \beta_{2} C O(g)+\beta_{3} N_{2}(g)+\beta_{4} H_{2}(g)$ & $(11)$ \\
\hline $\mathrm{CH}_{4}(g)+\frac{1}{2} \mathrm{O}_{2}(g) \rightarrow \mathrm{CO}(g)+2 \mathrm{H}_{2}(g)$ & $(12)$ \\
\hline $\mathrm{CH}_{4}(g)+2 \mathrm{O}_{2}(g) \rightarrow \mathrm{CO}_{2}(g)+2 \mathrm{H}_{2} \mathrm{O}(g)$ & $(13)$ \\
\hline $\mathrm{CH}_{4}(g)+\mathrm{CO}_{2}(g) \rightarrow 2 \mathrm{CO}(g)+2 \mathrm{H}_{2}(g)$ & $(14)$ \\
\hline $\mathrm{C}_{2} \mathrm{H}_{6}(g)+\mathrm{O}_{2}(g) \rightarrow 2 \mathrm{CO}(g)+3 \mathrm{H}_{2}(g)$ & $(15)$ \\
\hline $\mathrm{C}_{2} \mathrm{H}_{6}(g)+2 \mathrm{CO}_{2}(g) \rightarrow 4 \mathrm{CO}(g)+3 \mathrm{H}_{2}(g)$ & $(16)$ \\
\hline $\mathrm{C}_{3} \mathrm{H}_{8}(g)+1,5 \mathrm{O}_{2}(g) \rightarrow 3 \mathrm{CO}(g)+4 \mathrm{H}_{2}(g)$ & $(17)$ \\
\hline $\mathrm{C}_{3} \mathrm{H}_{8}(g)+3 \mathrm{CO}_{2}(g) \rightarrow 6 \mathrm{CO}(g)+4 \mathrm{H}_{2}(g)$ & $(18)$ \\
\hline $\mathrm{C}_{4} \mathrm{H}_{10}(g)+4 \mathrm{CO}_{2}(g) \rightarrow 8 \mathrm{CO}(g)+5 \mathrm{H}_{2}(g)$ & $(19)$ \\
\hline $\mathrm{CO}(g)+\frac{1}{2} \mathrm{O}_{2}(g)=\mathrm{CO}_{2}(g)$ & $(20)$ \\
\hline $\mathrm{H}_{2}(g)+\frac{1}{2} \mathrm{O}_{2}(g)=\mathrm{H}_{2} \mathrm{O}(g)$ & $(21)$ \\
\hline $\mathrm{H}_{2} \mathrm{O}(v)=\mathrm{H}_{2} \mathrm{O}($ condensado $)$ & $(22)$ \\
\hline $2\left(\mathrm{C}_{3} \mathrm{H}_{8}\right)(\mathrm{g})+6\left(\mathrm{H}_{2} \mathrm{O}\right)(\mathrm{v}) \Rightarrow 3 \mathrm{CH}_{3} \mathrm{COOH}($ condensado $)+8 \mathrm{H}_{2}(\mathrm{~g})$ & $(23)$ \\
\hline $3\left(C_{4} H_{10}\right)(g) \Rightarrow C_{12} H_{22}($ condensado $)+4 H_{2 .}(g)$ & $(24)$ \\
\hline
\end{tabular}

* Contribuição técnica ao 44 Seminário de Redução de Minério de Ferro e Matérias-primas, 15ㅇ Simpósio Brasileiro de Minério de Ferro e 20 ${ }_{8}^{\circ}$ Simpósio Brasileiro de Aglomeração de Minério de Ferro, 15 a 18 de setembro de 2014, Belo Horizonte, MG, Brasil. 


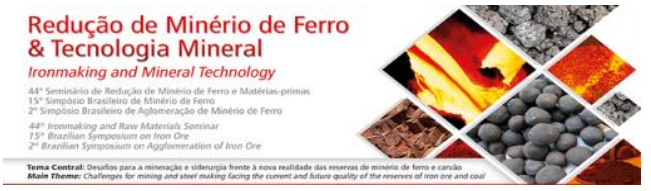

\subsection{Implementação Computacional}

As equações de transporte que descrevem o modelo multifásico e multicomponente foram resolvidas através da discretização usando a técnica de volumes finitos implícito, conforme descrita por Patankar [7]. O domínio compreende o volume do forno, sendo suas paredes descritas através de coeficientes de trocas térmicas baseado no modelo de resistências em série. O domínio foi discretizado em 150x20x20 volumes de controle e o intervalo de tempo de 60s, estes parâmetros foram adotados devido a grande dimensão do forno e o extenso período de fabricação. Esta discretização foi escolhida após refinamentos sucessivos que indicou não haver ganho significativo de acurácia para refinamentos posteriores erro global inferior a $3 \%$ ). $O$ escoamento gasoso foi resolvido através do acoplamento pressão velocidade utilizando-se o algoritmo SIMPLE enquanto o movimento do sólido (degradação da madeira) no interior do forno foi resolvido utilizando-se a conservação de massa e mudança de volume do material conforme as reações avançam. A solução transiente do movimento da carga sólida no interior do forno se faz necessária devido a mudança significativa de densidade do material remanescente e acomodações da carga no interior do forno, o que impõe o movimento induzido por forças de empuxo e gravitacionais. As demais equações do modelo representam a formação de líquidos, espécies gasosas e energia, sendo resolvidas de forma acoplada à cinética química. O modelo é resolvido de forma iterativa para tratar as não linearidades das equações de cinética, transferência de calor e momentum Equações (1) a (4) e equações para as taxas representando as cinéticas das reações listadas Equações (7) a (24) .

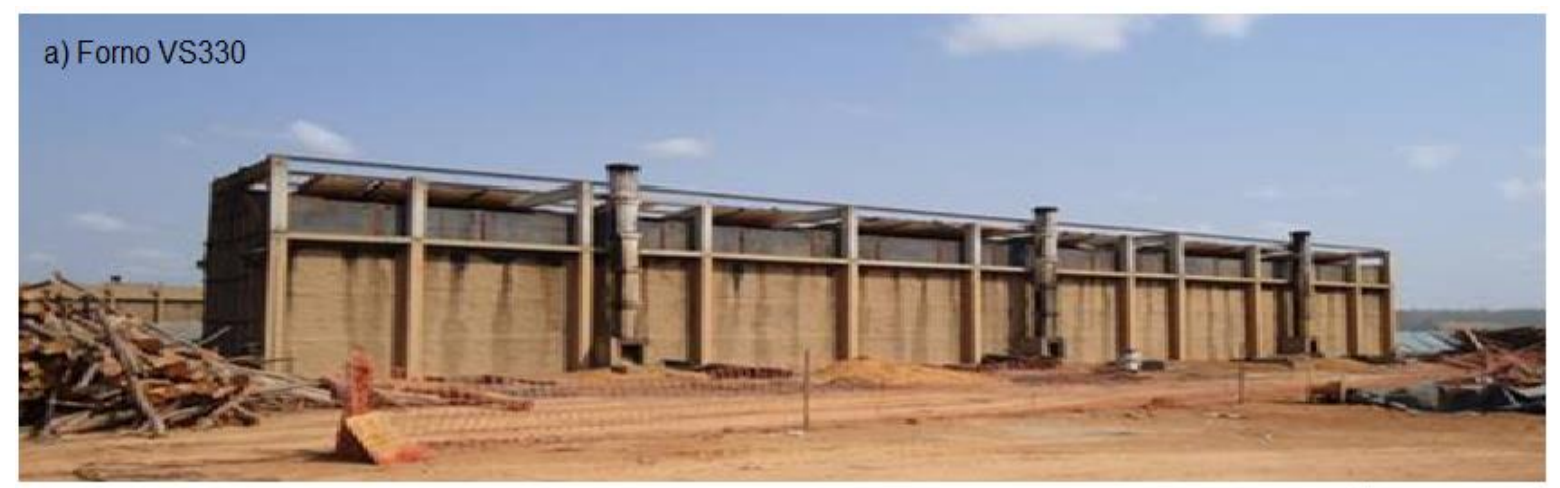

b) Modelo geométrico VS330

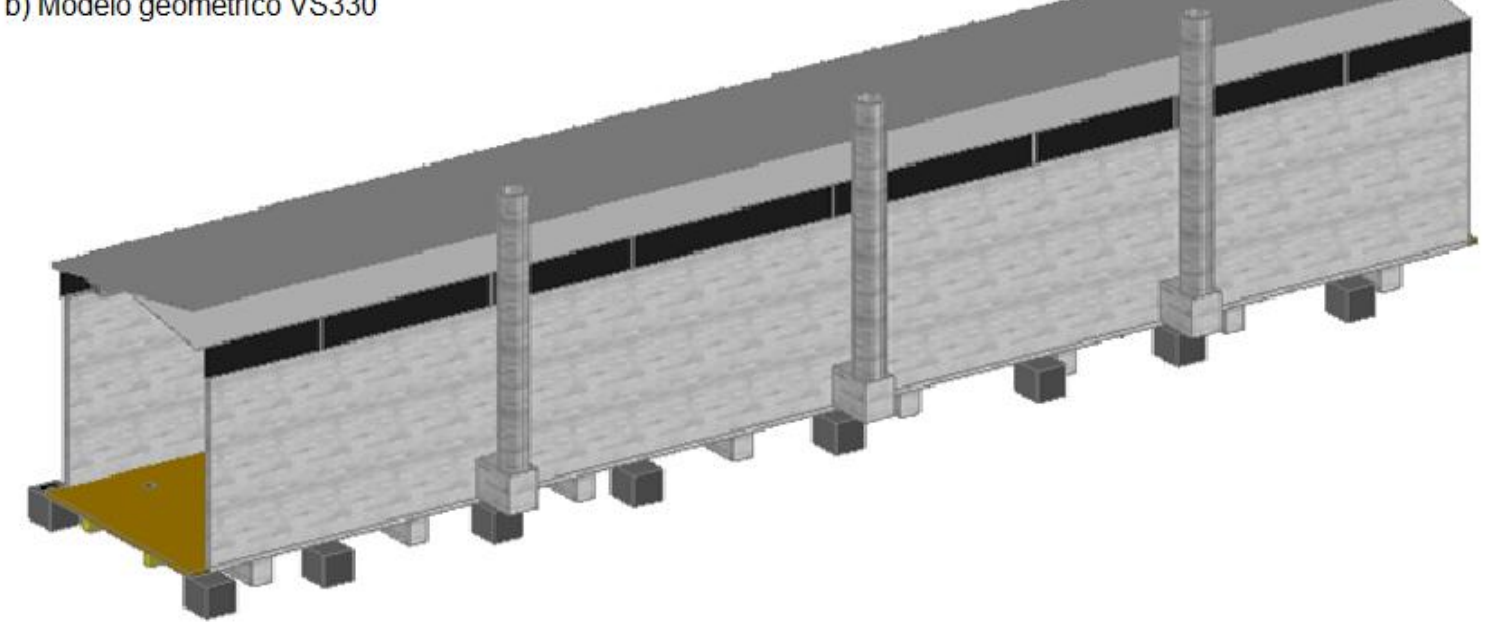

Figura 1. Forno construído com painéis de cobertura metálica e correspondente modelo geométrico para construção da malha numérica

* Contribuição técnica ao 44 Seminário de Redução de Minério de Ferro e Matérias-primas, $15^{\circ}$ Simpósio Brasileiro de Minério de Ferro e ${ }^{2}{ }_{85 \zeta b}$ Simpósio Brasileiro de Aglomeração de Minério de Ferro, 15 a 18 de setembro de 2014, Belo Horizonte, MG, Brasil. 
3 RESULTADOS E DISCUSSÃO

Neste estudo foram consideradas duas construções de fornos modelo VS330. No primeiro modelo o forno é construído em alvenaria, enquanto o segundo modelo é construído com base e lateral em alvenaria e copa recoberta com painéis articulados de manta isolante com placas metálicas que nas etapas de aquecimento, secagem e pirólise trabalham fechadas e minimizam perdas térmicas permitindo melhor aproveitamento de energia nas etapas endotérmicas. Na fase de resfriamento, os painéis são abertos formando um túnel de convecção induzido pela diferença de temperaturas, que promove a aceleração do processo natural de resfriamento pela copa do forno.

\subsection{Verificação do Modelo}

Com o objetivo de verificar o modelo desenvolvido temperaturas foram monitoradas em pontos distribuídos da superfície das portas de alimentação e desenfornamento e seus valores médios comparados com as previsões do modelo ao longo do tempo. Conforme observado na Figura 2(a) os valores previstos pelo modelo apresentam excelente concordância com aqueles monitorados. $O$ monitoramento das temperaturas, foram distribuídas ao longo da superfície da porta e medidos por pirômetro óptico de forma similar aos pontos considerados no modelo para calcular a média de temperaturas ao longo do tempo. As comparações foram realizadas através dos valores médios, definindo-os como representativos do estado interno do forno e são usados na pratica como indicativos operacionais para se identificar as fases do processo. Na Figura 2(a) também se apresenta uma comparação da evolução das temperaturas médias para os modelos de alvenaria e com painéis articulados, onde pode-se observar que nas etapas de pirólise se obtêm temperaturas maiores para os fornos de painéis articulados devido às menores perdas térmicas, promovendo a aceleração da etapa de pirólise, com consequente diminuição do consumo total de energia e aumento do rendimento gravimétrico.
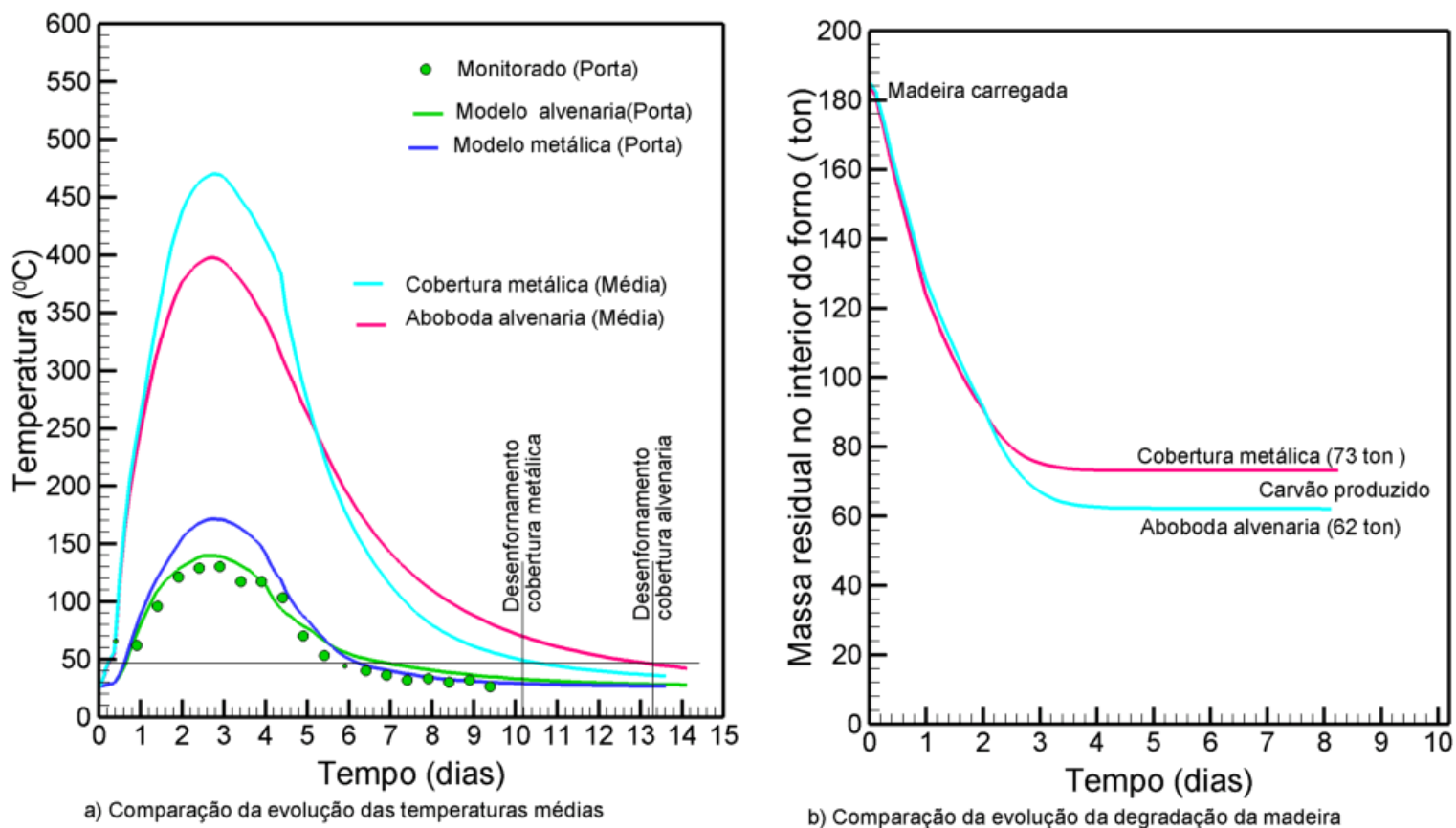

Figura 2. Verificação do modelo para a condição atual de operação dos fornos

* Contribuição técnica ao 44ํㅗㄴ Seminário de Redução de Minério de Ferro e Matérias-primas, $15^{\circ}$ Simpósio Brasileiro de Minério de Ferro e ${ }^{2}{ }_{85}$ Simpósio Brasileiro de Aglomeração de Minério de Ferro, 15 a 18 de setembro de 2014, Belo Horizonte, MG, Brasil. 

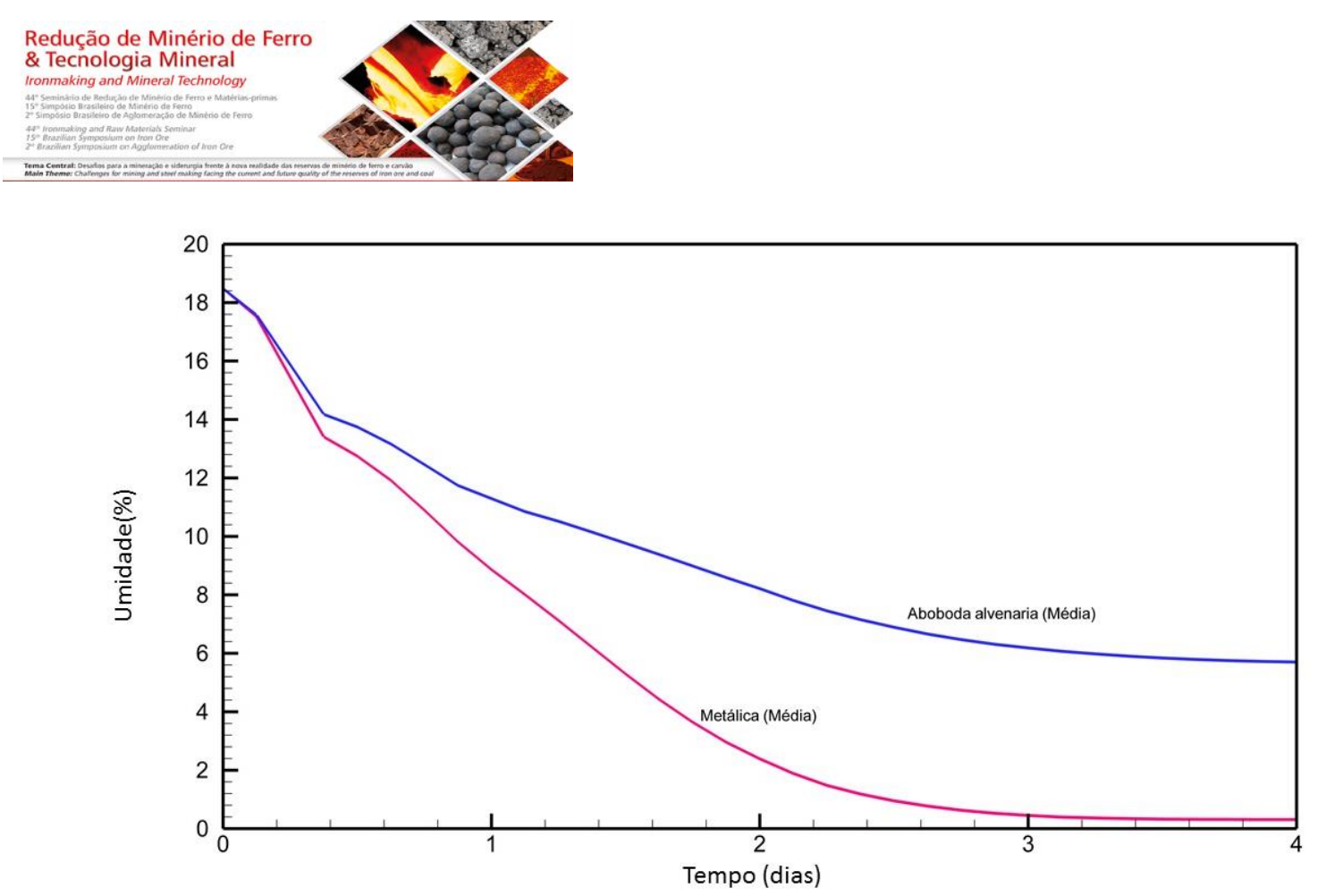

Figura 3. Evolução da fase de secagem da madeira $x$ tempo (h) para início da carbonização.

$\mathrm{Na}$ figura 2(b) apresenta-se um resultado típico da evolução da massa residual no interior do forno (carvão vegetal) mostrando que o rendimento é da ordem de $40 \%$ em massa, para o forno de cobertura metálica, para o presente caso o valor medido em campo foi de $36 \%$, mostrando que o modelo é capaz de prever com boa precisão o rendimento do processo, complementando a informação, para o forno de cobertura em alvenaria o rendimento é de $34 \%$.

A fase de secagem é apresentada através das curvas geradas no modelo, indicadas na Figura 3, onde observou-se uma redução significativa do tempo necessário para o forno com painéis articulados e consequentemente, através da redução da umidade residual, que permite otimizar o consumo energético do processo.

\subsection{Comparação da Evolução Interna dos Modelos com e sem Painéis Articulados}

Nesta secção as distribuições internas de temperaturas e escoamento gasoso são comparadas durante o processo de carvoejamento. Nas Figuras 4 (a) e (b), apresentam-se as distribuições internas de temperaturas e escoamento gasoso para o estágio inicial, que compreende o aquecimento e secagem da madeira. Observase que para um mesmo instante após o acendimento, o forno com painéis articulados apresenta-se numa condição térmica avançada em comparação ao convencional, possibilitando melhor desempenho do mesmo.

$\mathrm{Na}$ Figura 5, apresentam-se as condições internas de temperaturas para o instante final da etapa de secagem e inicial da pirólise. Observam-se para os dois modelos uma distribuição heterogênea de temperaturas devido às regiões onde ocorre combustão de madeira e consequente fornecimento de calor e frentes de secagem e diversas reações endotérmicas. Na Figura 6, mostra a etapa intermediária de aquecimento e secagem e início de pirólise para o forno padrão de alvenaria e com painéis articulados metálicos.

Este fenômeno contribui para acelerar o resfriamento através da promoção de uma recirculação interna do gás, devido aos efeitos de gradientes térmicos e consequente ação do empuxo associado ao incremento do coeficiente de convecção externo, devido à geração de um escoamento externo induzido, pela diferença de

* Contribuição técnica ao 44ํ Seminário de Redução de Minério de Ferro e Matérias-primas, 15o Simpósio Brasileiro de Minério de Ferro e 20 ${ }^{\circ}$ Simpósio Brasileiro de Aglomeração de Minério de Ferro, 15 a 18 de setembro de 2014, Belo Horizonte, MG, Brasil. 
temperaturas formando um efeito de túnel sob os painéis abertos. Estes efeitos podem ser claramente identificados na Figura 6 (b).

A comparação entre os resultados simulados para os fornos de alvenaria VS330 com a inovação dos painéis articulados mostra-se, portanto, que esta tecnologia é viável e proporciona um ganho no tempo total de produção do carvão vegetal de aproximadamente 5 dias, considerando abertura do forno aproximadamente a $45^{\circ} \mathrm{C}$, como apresentado na Figura 2(a)

Resultados de campo utilizando o protótipo deste forno apresentaram resultados similares, corroborando os resultados de simulação. Pode-se neste sentido afirmar que o modelo se mostra confiável para se estudar otimizações do ciclo de produção identificando-se oportunidades de melhorias futuras também nas etapas de pirólise, bem como verificar os impactos de novas práticas operacionais na qualidade e rendimento do processo. Conforme previsões do modelo apresentado na Figura 7, observa-se que a fixação do carbono também é aumentada da ordem de $5 \%$, devido ao processo de pirólise ocorrer a maiores temperaturas com menores perdas térmicas, quando comparada as duas curvas.

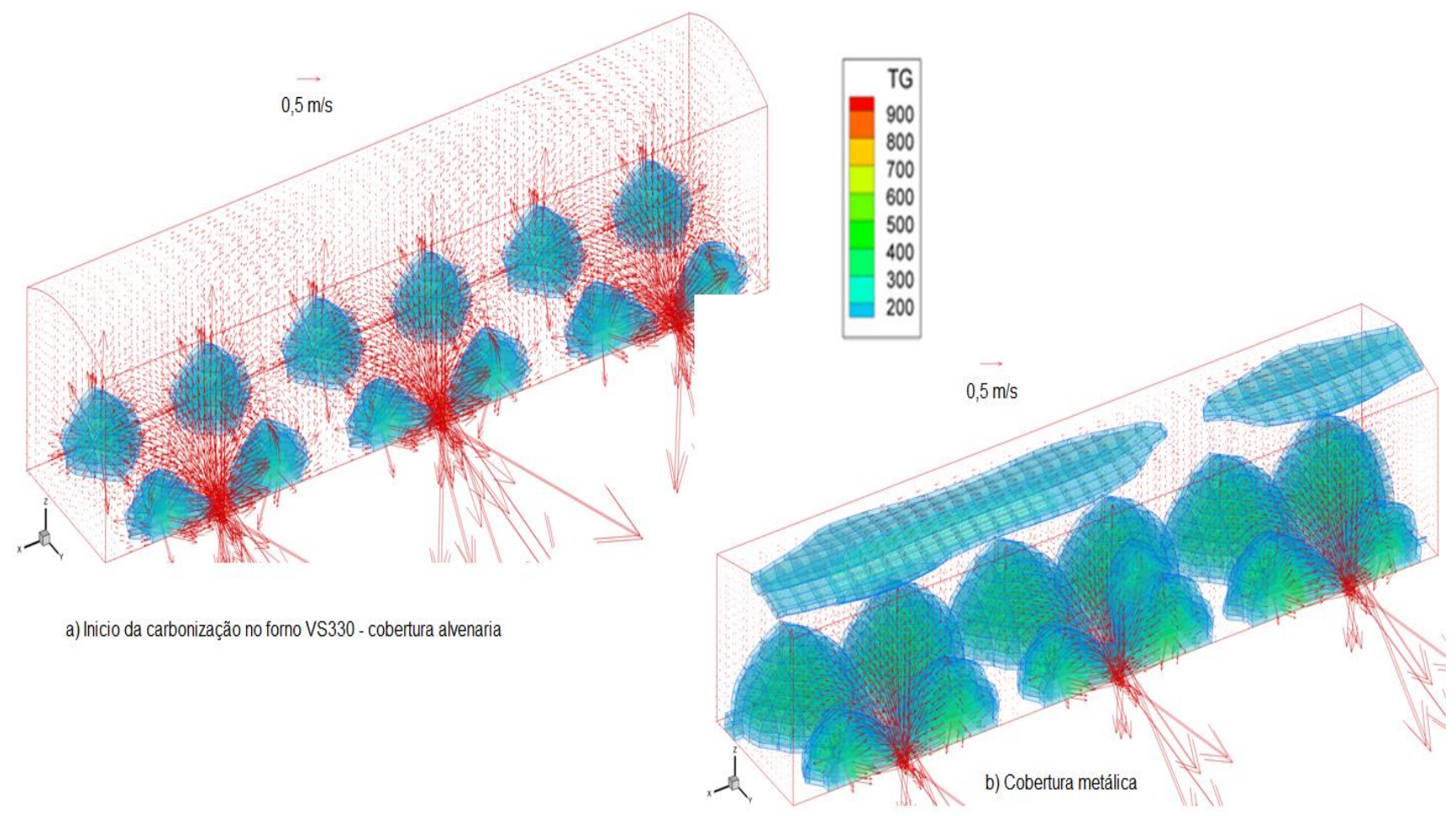

Figura 4. Comparação da etapa inicial de aquecimento e secagem para o forno padrão de alvenaria e com painéis articulados metálicos.

* Contribuição técnica ao 44ํㅗㄴ Seminário de Redução de Minério de Ferro e Matérias-primas,

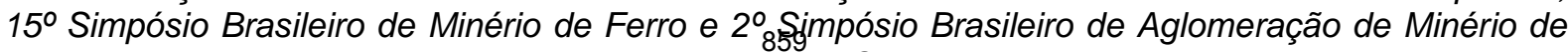
Ferro, 15 a 18 de setembro de 2014, Belo Horizonte, MG, Brasil. 

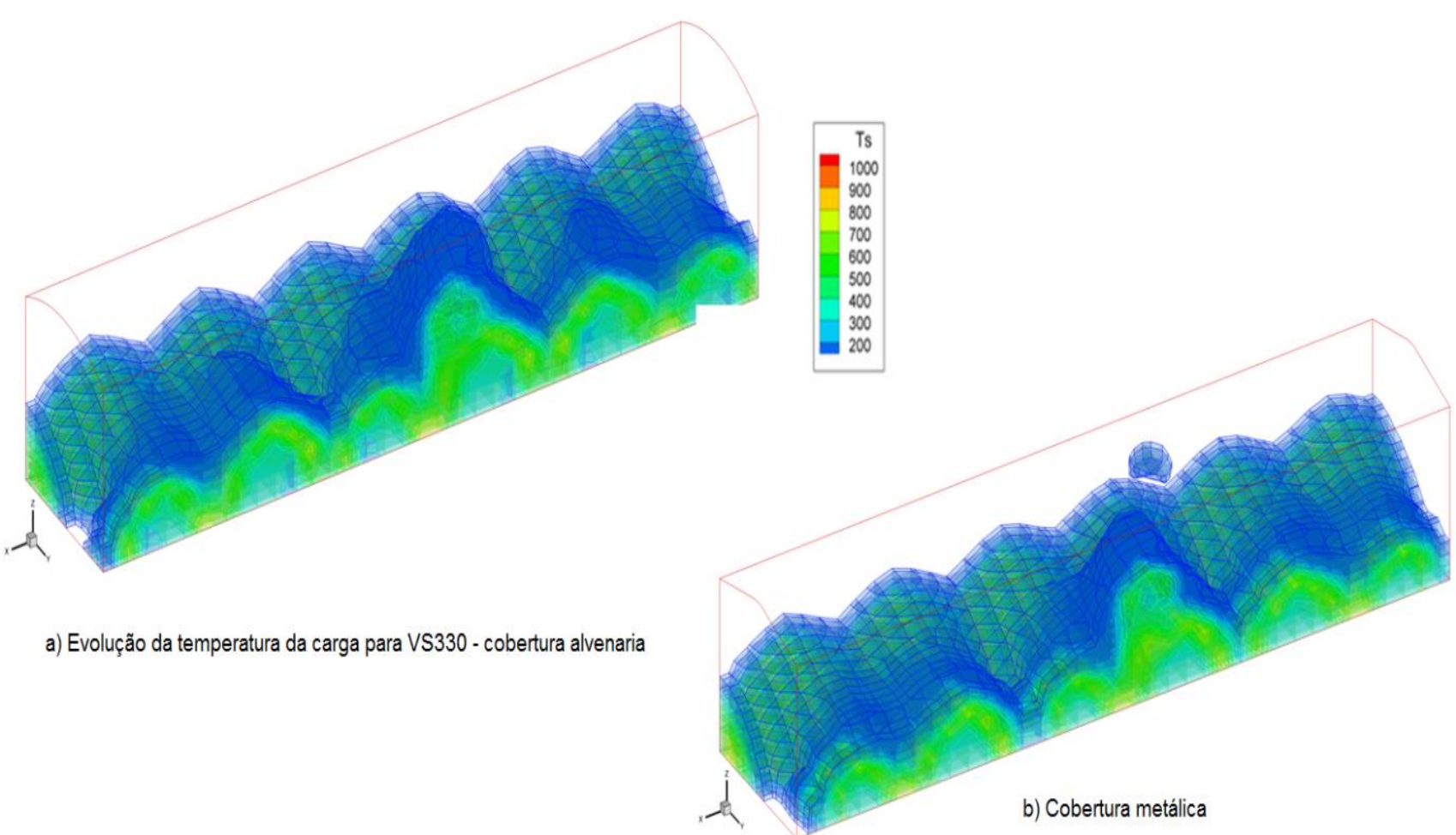

a) Evolução da temperatura da carga para VS330 - cobertura alvenaria

Figura 5. Comparação da etapa intermediária de aquecimento e secagem e inicio de pirólise para o forno padrão de alvenaria e com painéis articulados metálicos.

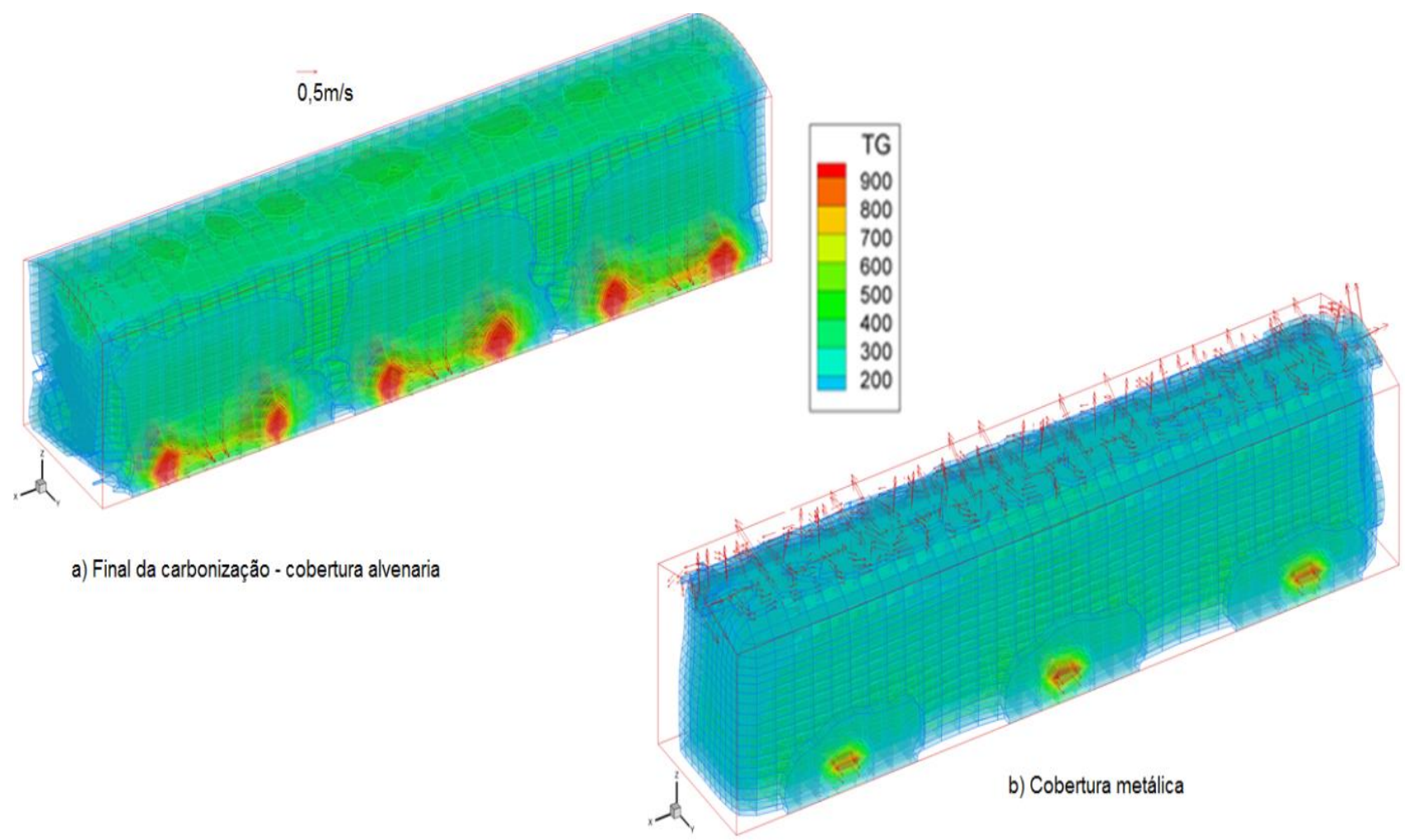

Figura 6. Comparação da etapa intermediária de aquecimento e secagem e inicio de pirólise para o forno padrão de alvenaria e com painéis articulados metálicos.

* Contribuição técnica ao 44 Seminário de Redução de Minério de Ferro e Matérias-primas, 15ㅇ Simpósio Brasileiro de Minério de Ferro e ${ }^{2}{ }^{\circ}$ SSimpósio Brasileiro de Aglomeração de Minério de Ferro, 15 a 18 de setembro de 2014, Belo Horizonte, MG, Brasil. 


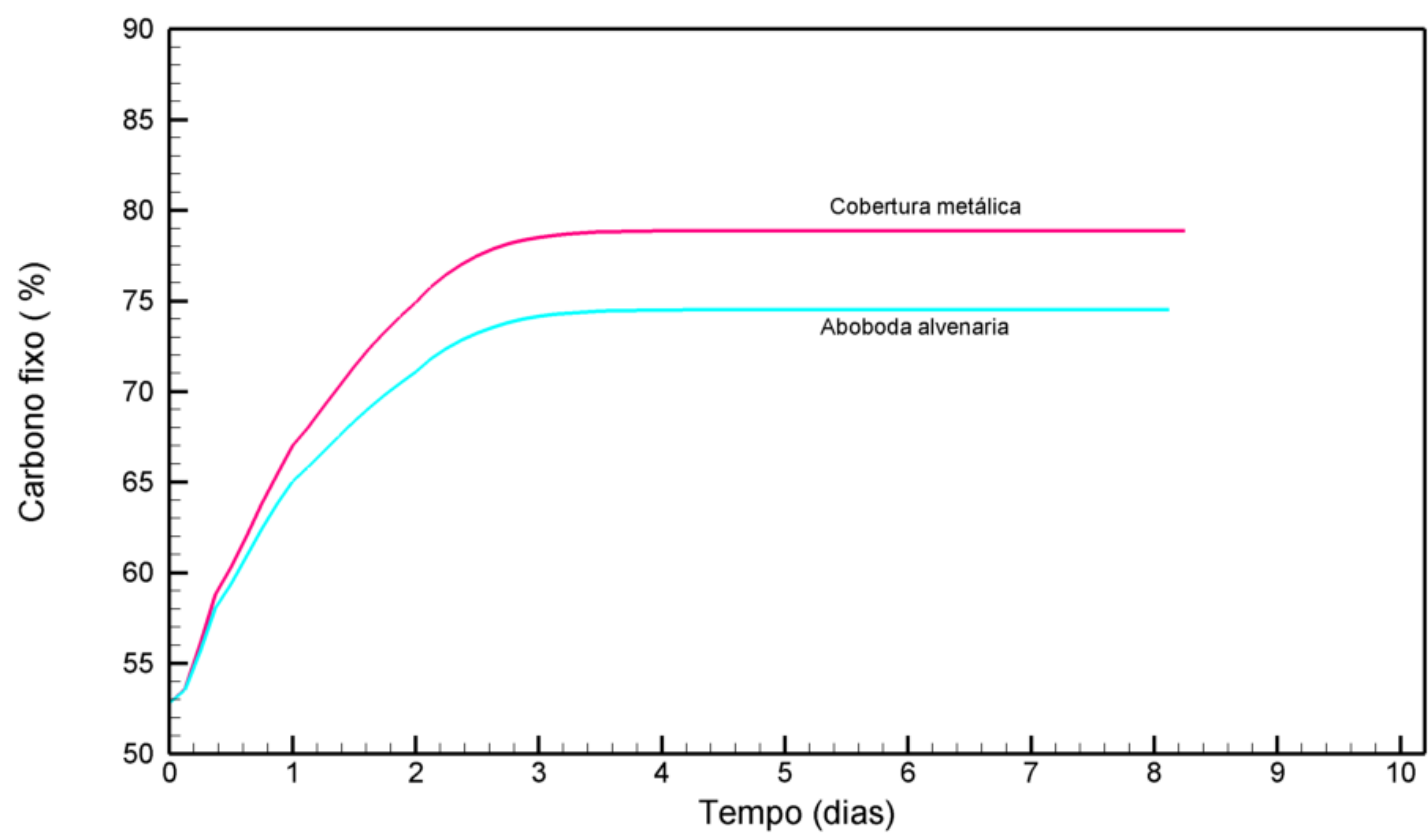

Figura 7. Comparação da evolução do carbono fixo médio no interior do forno VS330 em alvenaria e com painéis articulados.

\section{CONCLUSÕES}

O modelo foi validado, através dos dados coletados em campo e apresentou resultados satisfatórios dos fenômenos envolvidos ao longo das etapas de carvoejamento em fornos retangulares modelo VS330 em alvenaria e com cobertura metálica acoplado por painéis articulados, que utilizados em duas situações apoiados sobre o teto (fechados) do forno de cobertura metálica, nas fases de secagem e pirólise, e levantados (abertos), sobre o teto do forno, criando uma forte convecção do ar, com intuito de acelerar o resfriamento, o que proporcionou um ganho de até 5 dias no tempo total do processo de produção de carvão. $O$ modelo utiliza-se da formulação multifásica multicomponente, transiente e tridimensional. $\mathrm{O}$ modelo foi verificado através de comparações de previsões de temperaturas monitoradas cotidianamente no processo atual, bem como parâmetros globais do processo tais como rendimento mássico, tempo total de desenfornamento e ainda do volume de condensados gerados ao longo do processo. Com aplicação desta tecnologia, na construção do forno, o modelo mostrou um melhor o rendimento mássico do forno de cobertura metálica, quando comparado ao tradicional em alvenaria ou seja, com menor geração de cinzas devido a redução do tempo de exposição do carvão vegetal a alta temperatura, o que representa uma vantagem importante do ponto de vista de aproveitamento energético.

\section{Agradecimentos}

Os autores agradecem ao CNPq e FAPERJ pelo apoio e em especial ao Engenheiro Gilson Ricardo Truesmann (Votorantin Siderurgia) pelo apoio técnico, coleta de dados e discussões durante o desenvolvimento desta pesquisa.

* Contribuição técnica ao $44^{\circ}$ Seminário de Redução de Minério de Ferro e Matérias-primas, $15^{\circ}$ Simpósio Brasileiro de Minério de Ferro e ${ }^{\circ}{ }_{88}$ Simpósio Brasileiro de Aglomeração de Minério de Ferro, 15 a 18 de setembro de 2014, Belo Horizonte, MG, Brasil. 


\section{REFERÊNCIAS}

1 Raad TJ. Simulação do processo de secagem e carbonização do eucalyptus SPP. Tese de doutorado. Belo Horizonte: Universidade Federal de Minas Gerais - UFMG; 2004: 59-76.

2 Castro JA, Silva AJ, Sasaki Y, Yagi J. A six-phases 3D model to study Simultaneous injection of high rates of pulverized coal and charcoal into the blast furnace with oxygen enrichment. ISIJ International. 2011; 51(5): 748-758.

3 Aboyade OA, Carrier M, Meyers EL, Knoetze JH, Gorgens JF. Model fitting kinetics analysis and characterisation of the devolatilization of coal blends with corn and sugarcane residues. Thermochimica acta, 2012, 530(1):95-106.

4 Salazar CM, Coonor MA. Kinetic studies of the pyrolysis of wood with particular reference to eucalyptus regnans. in: Chemeca 83, paper 22b, the eleventh Australian Conference on Chemical Engineering , Brisbane,4-7,;N.S.W, 1983.

5 Fatch T, Roganne L, Richard J, Jabouille F. Modeling the thermal decomposition of a treated polywood from thermo-gravimetry and fourier transformed infrared spectroscopy experimental analysis. Journal of analytical and applied pyrolysis. 2013, 101(1):35-44.

6 Raad TJ, Pinheiro PC, Yoshida MI. Equação geral do mecanismo cinético da carbonização do eucalyptus SPP. Cerne, 2006, 12(2):93-106.

7 Patankar SV. Numerical heat transfer and fluid flow. (Hemisphere Series on Computational Methods in Mechanics and Thermal Science), CRC Press, Washington 1980.

* Contribuição técnica ao 44ํㅗㄴ Seminário de Redução de Minério de Ferro e Matérias-primas, 15ㅇ Simpósio Brasileiro de Minério de Ferro e ${ }^{2}{ }^{\circ}$ Simmpósio Brasileiro de Aglomeração de Minério de Ferro, 15 a 18 de setembro de 2014, Belo Horizonte, MG, Brasil. 